\title{
CONCEPCIÓN DE LA PERSONA Y SUS DERECHOS EN EL ANTEPROYECTO DE LA NUEVA CONSTTTUCIÓN ${ }^{1}$
}

Dr. Carlos Valderrama

A propósito del Proyecto de Nueva Constitución y de la suscripción del Pacto de Gobernabilidad han aflorado, como parte de la temática nacional nuevos conceptos introducidos por movimientos feministas y libre pensadores, que están contradiciendo los principios fundamentales sobre los que está construida nuestra Nación.

Por un lado se ignora juridicamente la condición natural del ser humano y, por el otro, se pretende invadir su intimidad.

En efecto, ciertos grupos con poder económico importante pretenden con algún éxito reducir al ser humano a la categoria de persona o de cosa, negando su realidad natural. Para comprender a cabalidad esta afirmación estimo oportuno precisar las diversas categorias que, en mi opinión, el Derecho puede concebir lo humano:

\section{Elser humano natural}

Esta categotia, y de la cual se ocupan diversas disciplinas, concibe al ser humano como elemento de la naturaleza. Al ser en sí mismo, despojado de

'Charla dictada en la Universidad Católica Sedes Sapientiae, en el marco del ciclo de conferencias del curso de Ética. Lus Olrvos, 15 de noviembre de 2002. 


\section{Carles Valderrama}

cualquier otra categoria que no sea producto de su propia realidad natural y espiritunl, considerado en su condición existencial. Es ese estado del cual habla J. Maritain en su obra Los derechos del bombre y la ley natural, dice Maritain: "Como no tengo tiempo de discutir tonterias (que siempre encuentran filósofos muy inteligentes para defenderlas brillanternente), supongo que admitis que existe una naturaleza humana, y que esa naturaleza es la misma en todos los hombres. Por otro parte, por tener una naturaleza, por estar constituido en una forma determinada el hombre tiene evidentemente fines que responden a una constitución natural y que son los mismos para todo"2. Hablamos, pues, en esta categoria de la naturaleza humana. De esa naturaleza humana que nos resulta tan evidente y que, en los últimos años, importantes pensadores se han esforzado en negar. Baste, como ejemplo de ello, uno de los pilares eu que sustenta Sartre su pensamiento del existencialismo moderno que tanto ha influenciado en los últimos años, especinlmente en el positivismo jurídico de Kelsen. Sartre en ese sentido afirma que: $\alpha E l$ hombre es libre y no hay ninguna naturaleza humana sobre la que se pueda apoyano ${ }^{3}$. Existe, pues, desde hace muchos años, una terca pretensión de negar lo evidente, de negar la dimensión humana del ser.

2. EJ ser humano como persona. En esta categoria, el ser humano es concebido desde su dimensión social. Cuando el ser entra en relación con otro ser surge la persona. En esta área el ser responde a su natural instinto de asociación, busca a través de un impulso espontáneo juntarse con otros seres para procurar su desarrollo y perpetuar su especie, este contacto supone un conjunto de relaciones a los que llamamos derechos y obligaciones. La persona humana es, pues, un ente imputable de derechos y obligaciones. Es producto de la historia y, por consiguiente, tiene una dimension cultural.

3. El ser humano concebido como cosa. Es una categoria que involucra una deformación del ser, pero es necesaria tenerla en cuenta en atención a la importante difusión gue ha tenido en la historia himana. En ella, el ser asurne la

\footnotetext{
"Marrtain 1., Los Derecbes del Honbre y he Loy Natumal, Buenos Aires, 1961, paig. 100

'Sartre J.P., Uexistencialisme ot nn bumanisme, Nagę, Pacis, 1967, pág. 52.
} 


\section{CONCEPCIÓN DE LA PERSONA Y SUS DERECHOS EN EL ANTEPROYECTO DE LA NUEVA CONSTITUCIONN}

condicion de elemento comercial, solo se aprecia desde el ámbito de la utilidad y el beneficio ajeno. La terminología moderna suele remitirse a ell como sel capital humano", usualmente pasa a ser un número en una estadística o en un balance. Es como la persona un producto de la cultura. Ejemplo de ello es la noticia que acaba de circular sobre la obesidad de las personas en Estados Unidos, que, según últimas estadísticas, llega al $36 \%$ de la población norteamericana, especialmente en niños. Dice la noticia que el presidente Bush ha puesta el grito en el cielo y ha ordenado una campaña intensa para reducir de peso a los nortcamericanos, continúa la noticia afirmando que la indignación del presidente se debe a que el problema de la obesidad implica un mayor gasto en el presupuesto de salud del gobierno ascendente a la suma de 5600 millones de dólares. Es claro, pues, que al gobierno norteamericano poco le importa la salud de sus ciudadanos, sino el mayor gasto presupuestal. La campaña está dirigida a reducir el gasto presupuestal estatal, y no a conseguir la buena salud de sus ciudadanos. La persona deja de tener valor en si misma y se convierte en un factor negativo dentro del presupuesto nacional de los Estados Unidos. Deja de valer como ser y se convierte en cosa.

Ahora bien, el derecho es a su vez producto de la cultura y ésta es producto de la historia del ser humano. El problema surge cuando eliminamos de la historia al ser humano y sólo lo concebimos como las personas o como cosas. Dicho en otras palabras, existe la tendencia actual en el derecho, de ignorar al ser humano en su dimensión natural y limitarlo únicamente a su esfera social y económica, es decir, solamente como persona y como cosa. Tal percepción genera una regulación juridica deformada que perjudica a la misma persona y a la sociedad en su conjunto, pues se ignora al individuo en su esencia básica. Esencia que es la que determina sus valores esenciales en su ser, estar, conocer, pensar, discernir, razonar, procrear, etc. primordiales, que están, además, por encima de la sociedad y del derecho, categorías que al ser ignoradas convierten a la sociedad y al derecho en fin de si mismos, en vez de ser medios para el adecuado desarrollo del ser humano.

Claro ejemplo de ello ha sido el Primer Anteproyecto de Reforma de la Constitución Peruana que, alegando consideraciones de forma, eliminó el primer artículo de la Constitución vigente, lo que en nuestra opinión, constituyó un proyecto de nueva constitución, que consagraba un desplazamiento de la persona 


\section{Carlos Valderrama}

humana como principio y fin de la sociedad y del Estado, sustituyendo, ademàs, el concepto de persona humana como ser natural por el de persona humana como ser cultural, lo que altera, sin lugar a dudas, la naturaleza esencial de la sociedad peruana, concebida asi desde su origen; pues una cosa es normar constitutivamente la sociedad como creación natural, y otra cosa es hacerlo bajo el presupuesto que la sociedad es producto del quehacer cultural humano, sin considerar su esencia primaria determinante.

En este sentido, nosotros entendimos que el Primer Proyecto de la nueva Constitución desplazaba a la persona humana como fin y principio de la sociedad peruana. Si bien la Comisión de Estudio de las Bases de la Reforma Constitucional del Perí habia propuesto restablecer el Preámbulo con que contaba la Constitución de 1979, como base doctrinal del nuevo texto constitucional, dicho Preámbulo no fue considerado en el Anteproyecto que se publicitaba y, muy por el contrario, tal Anteproyecto eliminó el Artículo 1." de la Constitución vigente, similar al texto del Articulo 1. de la Constitución de 1973, que consagra el príncipio de la supremacia de la persorra humana sobre cualquier otra categoria. El articulo que se pretendia suprimir textualmente dispone:

"Articulo 1. La defensa de la persona humaria y el respeto de su dignidad son el fin supremo de la sociedad y del Estadom

La razón que da la Comisión de Estudio de las Bases de la Reforma Constitucional del Perú para eliminar el referido Articula 1," es la siguiente:

(Las Constituciones del Perú no han contado con un titulo inicial que agrupe a los principios fundamentales que las orientan. En cambio, las últimas Constituciones de América Latina (Bolivia, Colombia, Paraguay, Brasil, Ecuador, Chile y Nicaragua) suelen contar con uno. Esto también ocurre con las constituciones europeas occidentales (Ėspana, Portugal, Alemania e Italia), así como con las nuevas constituciones curopeas orientales (Rusia, Bielorrusia, Polonia y las Repúblicas Bálticas) »y.

No encontramos xálida esta justificación. Setia menester hacer un análisis 


\section{CONCEPCIÖN DE LA PERSONA $Y$ SUS DERECHOS \\ EN EL. ANTEPROYECTO DE LA NUEVA CONSTITUCIÓN}

profundo sobre la estructura y naturaleza de tales constituciones para justificar en la nuestra un cambio de esa naturaleza. Además, lo foráneo no necesariamente tiene que ser bueno para nosotros: el Perú debe tener una realidad jurídica que responda a su propia identidad y que no es la misma que, por ejemplo, tiene Bielorrusia y las Repúblicas Bălticas. En todo caso, la Constitución vigente no está ajena a «tales modernismos».

Afortunadamente pudimos intervenir a tiempo y logramos que en el Pleno del Congreso se diera marcha atrás en esta pretensión y se repusiera en el Anteproyecto de Constitución el texto referido del Articulo 1." de la actual Constitución.

Explicando un ejemplo sobre la manera con la que en nuestro país se pretende ignorar juridicamente la condición natural del ser humano, con todas las consecuencias que ello traeria, como definir culturalmente el principio y fin de la vida humana, conviene ahora referirnos a la manera en que se pretende invadir legaimente la privacidad de las personas. Para ello recurriremos nuevamente al Primer Anteproyecto de Ley Modificadora de la Constitución aprobado por la Comisión de Constitución del Congreso de la República.

En efecto, el proyecto referido subtitula el Artículo 3." como de «Libertad de Conciencias y bajo este título trata la libertad de conciencia, opinión y religión. Evidentemente, tal titulación induce a confusión, pues podria dat a entender que son la misma cosa o que la opinión y la religión son categorias de la conciencia. Inducir a una confusión de esta naturaleza es grave, pues estas tres categorias independientes en su objetivo, constituyen, las tres a la vez la frontera de la intimidad de la persona humana. Delinean ese espacio en que la persona es lo que es, única e irrepetible. Su meçanismo de interacción le otorga la humanidad al ser. La opinión como expresión del pensamiento, y la religión como expresión de la creencia son los que informan a la conciencia para que ésta pueda emitir su juicio moral sobre el bien y el mal. El pensamiento está dirigido a determinar lo verdadero de lo falso, y la creencia lo sacro de lo profano. Insinuar categorías de una sobre la otra, como lo hace el proyecto de la Nueva Coristitución, constituye una grave deformación del ser natural de la persona humana. Es más, como 


\section{Carlos Valderrama}

veremos mas adelante, se pretende regular normativamente la acción de la libertad de conciencia mediante una ley orgánica, y si como suponemos, por lo antes dicho, que el pensamiento y la religión constituirian categorias de la conciencia, se estaria previendo la posibilidad de regular por ley el ser intrinseco de la persona, con lo que se estaria facilitando a gobiernos inescrupulosos instautar la más rerrable de las dictaduras; incluso se podria llegar a un fundamentalismo agnóstico insoportable.

El últumo párrafo del Artículo 3." del Anteproyecto de Nueva Constitución, antes citado, establece: ‘Se reconoce el derecho a la objeción de conciencia, cuyos alcances se desarrollarán por ley orgánicay. Esta norma merece de nuestra parte, como señalamos antes, el comentario siguiente:

El reconocimiento al derecho de la objeción de conciencia es innecesario, pues este se encuentra implicito de manera irrefutable en el contenido de la libertad de conciencia, ya declarado por el Proyecto de Nueva Constitución. En efecto, la libertad de conciencia se hace realidad a través de una doble dimensión. Por un lado, el libre derecho a la formación de la conciencia y, por el otro, el derecho a la objeción de conciencia.

La objeción de conciencia consiste en el derecho de resistencia personal a una prescripción jurídica, por ser ésta contraria a una prescripción moral que se considera prevalente. Se trata de un conflicto subjetivo entre deber jurídico y deber moral. Es la negativa, por motivos de conciencia, a realizar un acto o conducta que en principio resultaria juridicamente exigible. Se sustenta en el valor prioritario de la persona frente al Estado.

Obviamente, la objeción de conciencia pierde eficacia cuando la persona carece de un valor prioritario frente al Estado, como lo hace el presente Proyecto de Nueva Constitución al suprimir el Artículo 1. de la actual Constitución. Tal perdida de prioridad faculta y facilita al Estado para poder definir los alcances de la objeción de conciencia, via una ley o norma, como lo hace este último párrafo comentado, sometiendo la conciencia de la persona al poder del Estado. Ya en el Articulo 3." del proyecto se evidencian los graves inconvenientes de desplazat a la 


\section{CONCEPCIÓN DE LA PERSONA Y SUS DERECHOS \\ EN EL ANTEPROYECTO DE LA NUEVA CONSTITUCIŌN}

persona humana como fin y principio de la sociedad y del Estado, Desplazamiento que los autores del Proyecto denominan imodernos,

La objeción de conciencia no puede ser regulada por una norma, pot más korgánicau que pretenda ser ésta:

- Porque como queda dicho se trata de un conflicto entre el deber moral y el deber jurídico, es decir, como en toda confrontación, estả por un lado el mandato de la norma moral y frente a ella, por el otro, el mandato de una norma juridica, confrontación en la que una de ellas no puede ser juez y parte; es decir, existe imposibilidad real de que sea una de las partes en conflicto, en este caso la norma jurídica, la que determine cuándo y de qué manera se confronta o si es posible o no tal confrontación. Ello constituye un despropósito intolerable.

- El deber moral siempre es y será un acto de la intimidad de la persona, dentro del cual la norma juridica no tiene competencia; en consecuencia, se encuentra imposibilitada de determinar ets qué momento y bajo qué condiciones se produce la confrontación entre lo moral y lo juridico, y menos aún los términos en que deba resolverse tal confrontación.

- A mayor abundamiento, la confrontación moral que da origen a la objeción de conciencia es siempre individual, nunca colectiva, por lo que no puede ser normada por una ley general, ya que seria imposible prever todas las individualidades de todos los ciudadanos en un número infinito de situaciones específicas particulares. Existe, pues, un imposible juridico.

- Por último, como queda dicho, incurrir en normar el lado íntimo y espiritual de la persona abre las puertas al poder de turno de un control total del ser humano.

La doctrina juridica internacionalmente aceptada en esta materia, por las razones expuestas, se inclina por establecer la necesidad de una norma de la más alta categoría que se limite a reconocer y definir, como valor supremo en la 


\section{Carlos Valderrama}

sociedad, el derecho a la libertad de conciencia, dejando a los tribunales, que conocen de individualidades, resolver los conflictos que se presenten entre un deber moral y un deber juridico.

Existen, en nuestro pais, experiencias pasadas en virtud a la cual el Estado, baciendo uso de su poder normativo, prohibió a los facultativos de la salud ejercer su detecho a la objeción de conciencia, cuando se trataba de programas de planificación familiar (sahud reproductiva), que contenian acciones de esterilización y de aborto.

\section{Estado pluricultural}

Llama tambièn nuestra atención la incorporación en el Articulo 85 del Anteproyecto de Nueva Constitución del término spluriculturals al redefinir al Perá. En efecto, ninguna otra Constitución antetior, desde la de 1826, ha definido al Peni aludiendo a su calidad cultural. Es, pues, una novedad cuya inclusión en el texto constitutivo traerá, según entendemos, serias dificultades y conflictos. No hemos encontrado en los textos correspondientes ni en diccionario o cnciclopedia alguna, la definición del término pluricultural, por lo que recurriremos a los elementos que la conforman: pluralidad y cultura.

\section{Por plualidad, a la calidad de ser más de uno.}

Cultura, como la entidad que surge como resultante de un proceso histórico que originan una teligión, una moral, un lenguaje, una tradición que involucra un modo de vida y costumbres, un grado de desarrollo artístico, científico e industrial, en una época y en un grupo social determinado

Si esto es así, debemos concluir que el proyecto de Constitución está definiendo al Perú como la suma diferenciable de más de un proceso histórico, más de una tradición, más de un grado de desarrollo, más de una época, y son varios los grupos sociales determinados. Es decir, el Perú carecería de unidad. En este sentida, la pluriculturidad desconoceria la existencia de un proceso histórico 


\section{CONCEPCION DE LA PERSONA Y SUS DERECHOS EN EL ANTEPROYECTO DE LA NUEVA CONSTITUCIÓN}

y cultural que ha forjado al Perí como nación; la exaltación de expresiones culturales parciales podrian ser contrapuestas a una visión de conjunto.

La pluralidad de cultura implica una reinterpretación del Perí y sus fundamentos, como la tradición, su historia, su motal y su religión, realizada desde la óptica particular de cada una de las culturas que integran esa gran pluralidad. Es decir, el Perú sumergido conceptualmente, en un relativismo absoluto, por propia declaración de su constítución. Se pierde el sentido objetivo de la unidad, consagrándose la posibilidad de un libertinaje de acción, pues cada quien actuaría de acuerdo a los dictados de su propia cultura, dentro de un mismo territorio geográfico; seria imposible establecer normas de gobierno uniformes para todas, habtia que legislar para cada cultura en particular, lo que nos conduciría a la anarquia.

Por ejemplo, si constitucionalmente se reconoce a la Iglesia Católica como un elemento importante en la formación histórica, cultural y moral del Pení, tal reconocimiento solo abarcaría a una de las culturas en que la pluralidad Perí consiste. De tal suerte, que será válido sostener un tipo de moral diferente a la consagrada por el mundo cristiano occidental, con lo que además rela tivizamos el concep to de lo que es el rorden públicon, la moral pública y las buenas costumbres.

Por último, si el contenido de la identidad nacional es ia cultura de un pueblo, y si gracias a la identidad se nos identifica como unidad social, histótica y juridica, estariamos constitucionalmente negando la existencia de una propia identudad, si reconocemos la convivencia de varias culturas en una misma categoria juridica denominada Perú.

Nosotros creemos que el Perú es una sola cultura heterogénea, es decir, una sola realidad cultural compuesta de varios elementos. La cultura peruana es metafóricamente como el rio Amazonas, formado por dos grandes rios y nutrido por muchas fuentes y diversos rios, con muchos colores de agua, caudales y velocidades en el movimiento, en que además de tener un mismo cauce, sus diferentes elementos tienen contactos e interacciones. Avanzan juntos hacia un mismo destino y forman an solo ríb. Muy distinto es entender al Perú como una 


\section{Carlos Valderrama}

gran cuenca geográfica, con tios diferentes que no se entrelazan, que avanzan por sus propios cauces, no llegando nunca a integrar una gran unidad.

No es lo mismo pluticultura que cultura heterogénea; nosotros, el Perú, somos una sola realidad histórica, con un mismo pasado, con un presente común y con la misma esperanza de un futuro mejor, aunque estemos formados de diversos "colores, velocidades y caudales".

¿Por qué hacer énfasis en la fragmentación y no mencionar los lazos unificadores? Somos como una tierra parda, con pinceladas de color negro, amarillo y blanco, a la que le cuesta un trabajo enorme definir sus contornos propios. ¿Por qué el afán de interrumpir nuestro trabajo de procurat nuestra propia identidad, quebrándonos y dividiéndonos con una declaración constitucional de pluriculturalismo que tanto daño nos hace en la búsqueda de nuestra madura unidad?. Baste aquí repetir las sabias palabras del maestro Basadre en su magna obra la Historia del Perú Republicano: «EI Perú es ... a pesar de todos los esfuerzos, una inmensa tarea por hacer; $y$, a pesar de todas las realizaciones, una bella promesa aún no cumplidan.

\section{La ideología de género}

También llama la atención cómo el Anteproyecto de Reforma Constitucional introduce en distintos artículos el término rgéneron, reemplazando con él a la palabra «sexo", como es el caso del articulo 1 inciso 2 que señala:

Art. 1. Toda persona tiene derecho:

$[\cdots]$

Inc. 2. A la igualdad. Está prohibida toda forma de discriminación por motivo de origen, filiación, raza, género, idioma, religión, opinión, condición económica, discapacidad o de cualquier otra índole, que tenga por objeto o por resultado anulat o menoscabar el reconocimiento, goce o ejercicio de los derechos de la personam 


\section{CONCEPCION DE LA PERSONA Y SUS DERECHOS \\ EN EL ANTEPROYECTO DE LA NUEVA CONSTITUCIÕN}

Oel artículo 8 que señala lo siguiente:

"Articulo 8. Extradición

Toda persona cuya extradición o entrega es solicitada tiene los derechos reconocidos en los tratados de los que el Perú es parte. No se concede la extradición si se considera que ha sido solicitada con el fin de perseguir o castigar por motivo de origen, raza, género, idioma, religión, opinión, condición económica a cualquier otra forma de discriminación-»

Asimismo, los articulos 27,34 y 47 :

"Artículo 27. Educaciốn intercultural

Es deber del Estado promover la educación intercultural, bilingüe, con equidad de género.n

"Articulo 34. No discriminación en educación.

El Estado asegura que nadie se vea impedido de recibir educación adecuada por razón de su condición social, económica, raza, color, género, idioma, religion o de cualquier otra índole,

"Articulo 47.

[...]

EI Estado debe asegurar que nadie se vea impedido de disfrutar de su derecho a la salud por razón de su condición social, económica, raza, color, gènero, idioma, religión o de cualquier otra indolen.

Como hemos indicado, los artículos en cuestión eliminan la palabra xsexon y lo cambian por ugéneron. ¿¿A quẻ se debe esta modificación de una palabra por otra?

¿Son sinónimas? Y, si lo fueran, ¿cuál seria la necesidad de hacer el cambio? De la investigación que realizamos hemos encontrado que la palabra kgéneron viene siendo ualizada actualmente con un contenido bastante distinto al de usexon, por una corriente denominada «Ideología de Género" a «Perspectiva de Génerow, que viene introduciendo su dideologias en las normas de muchos estados y organismos 


\section{Carlos Valderrama}

interancionales. ¿Este es el caso del Anteproyecto de Reforma Constitucional de nuestro pais? Para dilucidar esta interrogante consideramos relevante que previamente profundicemos en lo que es la «Ideologia de géneron, $y$ para ello hemos utilizado como referencia el documento La ideologia de género sus peligros y aloances. elaborado en 1998 por la Comisión Episcopal de Apostolado Laical de la Conferencia Episcopal Peruana en base al informe La desconstrucción de la mujer de Cale O'Leary.

\section{¿Qué es la ideologia de género?}

El término "género» no es una forma de decir "sexo" para evitar el sentido secundario que "sexo" tiene, sino que "género» se refiere a seres humanos masculinos y ferneninos, Lo cierto es que esto no es así: la ideología de género difunde toda una knueva perspectivan del término, esta perspectiva se refiere al término género como (croles socialmente construidos».

En el libro Gender Trowble: Feminism and the Subversion of Identity (El problema dol género: el feminismo y la subversión de la identidad) de Judith Butler, se señala:

«El género es una construcción cultural; por consiguiente no es ni resultado causal del sexo ni tan aparentemente fijo como el sexo... Al teorizat que el género es una construcción radicalmente independiente del sexo, el género mismo viene a ser un artificio libre de ataduras; en consecuencia hombre y masculino podrían significar tanto un cuerpo femenino como uno masculino; mujer y femenino, tanto un cuerpo masculino como uno femeninon.

Fue en la IV Conferencia Mundial de las Naciones Unidas sobre la Mujer, realizada en septiembre de 1995, en Pekín, donde se presentó esta nueva perspectiva. Es desde dicha cumbre que la uperspectiva de géneron ha venido buscando introducirse en diferentes ámbitos no sólo de los paises industrializados, sino además de los paises en vias de desarrollo.

Precisamente en la cumbre de Pekin se solicitó a sus principales propulsares una definición que pudiera aclarar el debate. Asi, la directiva de la conferencia de la ONU emitió la siguiente definición: 


\section{CONCEPCIONN DE LA PERSONA Y SUS DERECHOS \\ EN EL. ANTEPROYECTO DE LA NUEVA CONSTITUCIÕN}

"El género se refiere a las relaciones entre mujeres y hombres basadas en roles definidos socialmente que se asignan a uno u otro sexo".

Dicha definición fue completada en la misma cumbre de la siguiente maneta:

"El sentida del término "género" ha evolucionado, diferenciándose de la palabra "sexo" para expresar la realidad de que la situación y los roles de la mujer y del hombre son construcciones sociales sujetas a cambiom.

Con dicha intervención quedó aclarado que los partidarios de la perspectiva de género proponen que sno existe un hombre natural o una mujer natural, que no hay conjunción de características o de una conducta exclusiva de un sólo sexo, ni siquiera en la vida psiquicay. Así, «la inexistencia de una esencia femenina o masculina nos permite rechazar la supuesta "superioridad" de uno u otro sexo, y cuestionar en lo posible si existe una forma "natural" de sexualidad humanas. 